Supporting Information

\title{
Monodisperse Selectively Permeable Hydrogel Capsules Made from Single Emulsion Drops
}

Mathias Steinacher ${ }^{1}$, Alice Cont ${ }^{2}$, Huachuan Du ${ }^{1}$, Alexandre Persat ${ }^{2}$, Esther Amstad ${ }^{1 *}$

${ }^{1}$ Soft Materials Laboratory, Institute of Materials, École Polytechnique Fédérale de Lausanne (EPFL), 1015 Lausanne, Switzerland

${ }^{2}$ Institute of Bioengineering and Global Health Institute, School of Life Sciences, École Polytechnique Fédérale de Lausanne (EPFL), 1015 Lausanne, Switzerland

* esther.amstad@epfl.ch 


\section{Videos}

Movie S1: Video of hydrogel capsules filled with a few individual bacteria swimming in the liquid core of a capsule.

Movie S2: Hydrogel capsules containing magnetic iron oxide nanoparticles in their core, enabling an easy manipulation of the capsules. 


\section{Tuning the capsule size}
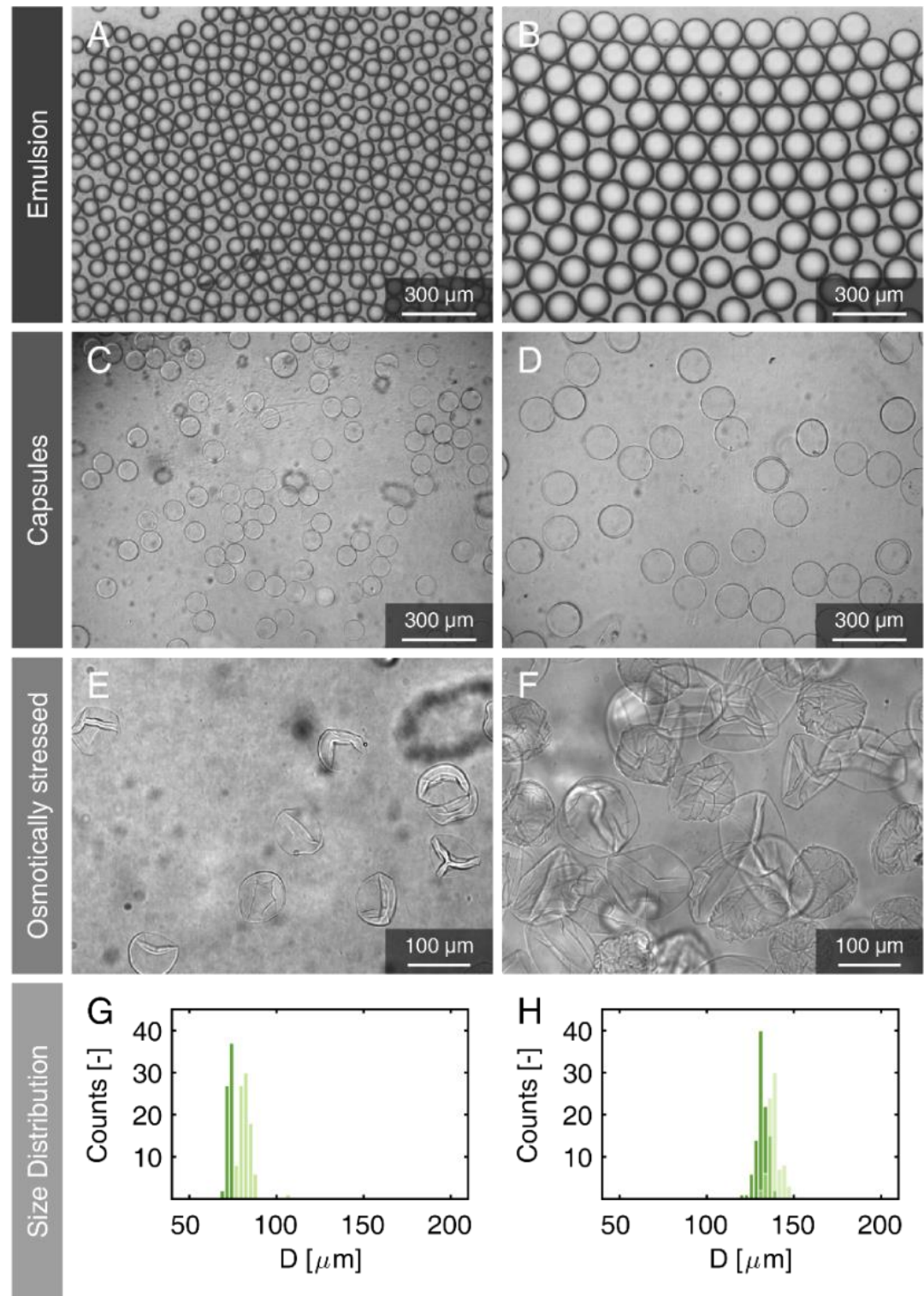

Figure S1. Fabrication of capsules with varying diameters. Optical micrographs of (A, B) drops and $(C, D)$ the resulting capsules. (A, B) Monodisperse water-in-oil single emulsions are produced in a microfluidic flow-focusing device by injecting the dispersed phase at a rate (A) $Q_{d}=0.3 \mathrm{ml}$ $\mathrm{h}^{-1}$ and the continuous phase at $\mathrm{Q}_{\mathrm{c}}=1 \mathrm{ml} \mathrm{h}^{-1}$, and (B) $\mathrm{Q}_{\mathrm{c}}=0.4 \mathrm{ml} \mathrm{h}^{-1}$ and $\mathrm{Q}_{\mathrm{d}}=0.3 \mathrm{ml} \mathrm{h}^{-1}$. Optical micrographs of $(\mathrm{C}, \mathrm{D})$ the capsules after UV crosslinking and transferring them in an aqueous 
phase and (E, F) osmotically stressing them by dispersing them in an aqueous solution containing $1 \mathrm{wt} \%$ PEG $20 \mathrm{kDa}$. (G, H): Size distribution of single emulsion drops (dark) and capsules (light).

\section{Capsule shell properties}

Dried hydrogel capsules show wrinkles that are characteristic for thin-walled capsules, as shown in Figure S2.

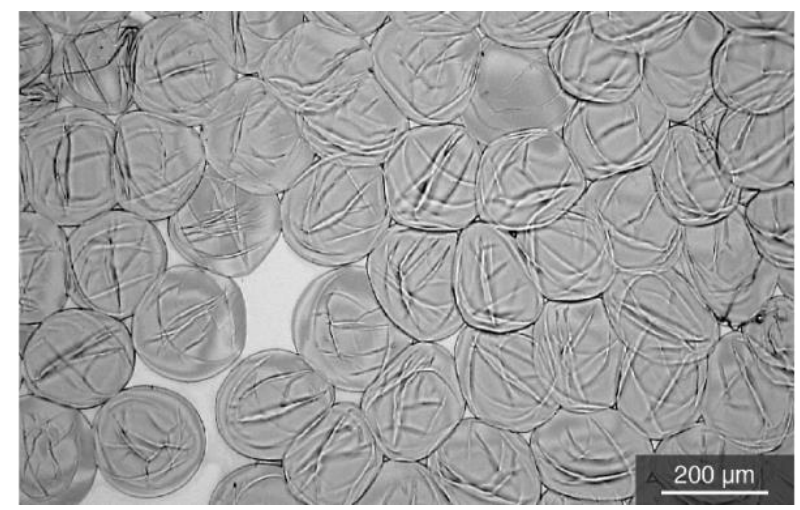

Figure S2. Optical micrograph of dried hydrogel capsules.

The Young's modulus of the hydrogel capsules is estimated by analyzing the deformation of osmotically stressed capsules. Optical micrographs of the emulsions and stressed capsules are shown in Figure S3. 
A

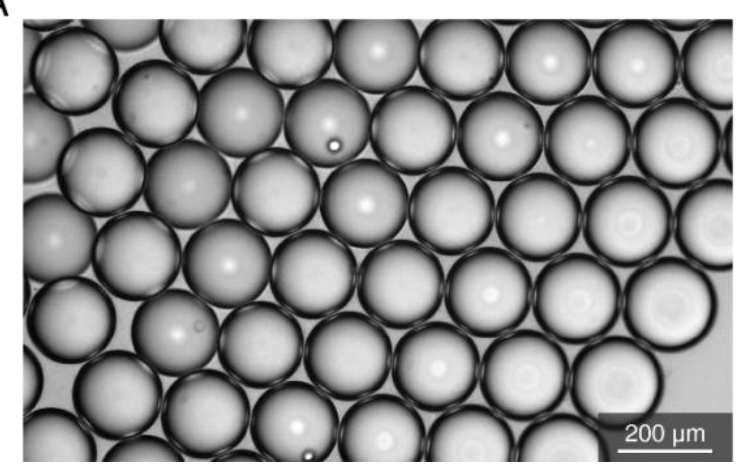

B

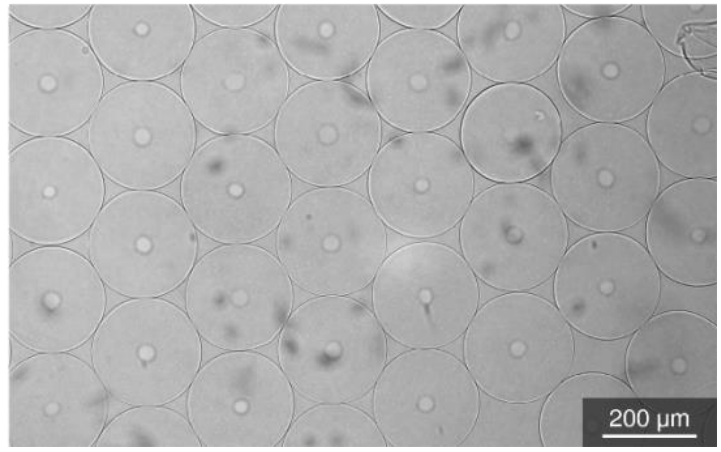

Figure S3. Optical micrograph of (A) an emulsion containing $0.5 \mathrm{wt} \%$ PDADMAC and (B) the resulting capsules dispersed in water.

The permeability of the hydrogel capsules is investigated by loading them with a fluorescently labelled dextran, as shown in Figure S4.

A

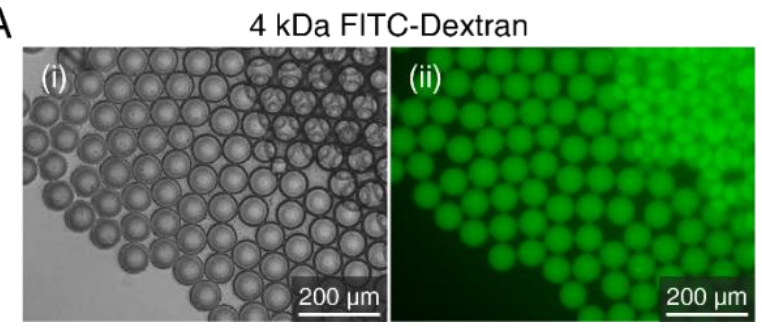

B

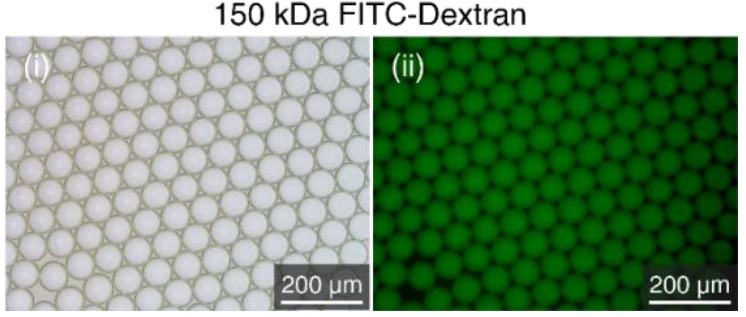

Figure S4. Quantification of the permeability of capsules. (i) Brightfield and (ii) fluorescence images of emulsion drops prior to polymerization loaded with FITC-labelled dextran with a molecular weight of (A) $4 \mathrm{kDa}$ and (B) $150 \mathrm{kDa}$. 


\section{Bacteria loaded capsules}

A

B
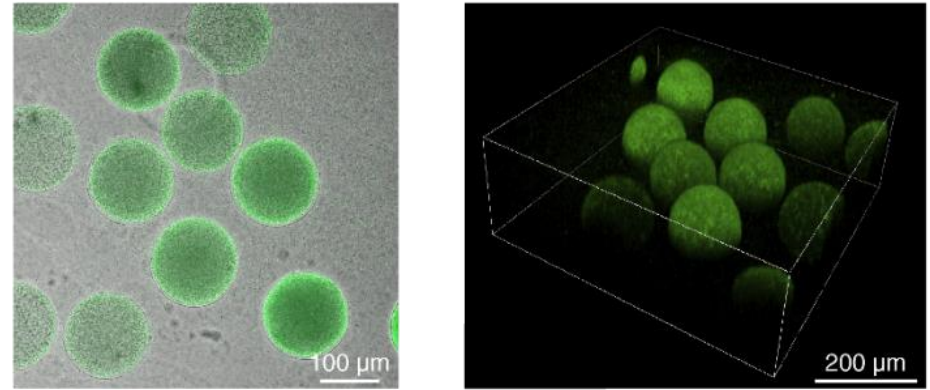

Figure S5. (A) Overlay of a brightfield and fluorescent images of multiple bacteria-containing capsules with (B) the 3D reconstruction obtained from a $\mathrm{z}$-stack after their incubation for $13 \mathrm{~h}$ at $37^{\circ} \mathrm{C}$.

\section{PAA loaded capsules}
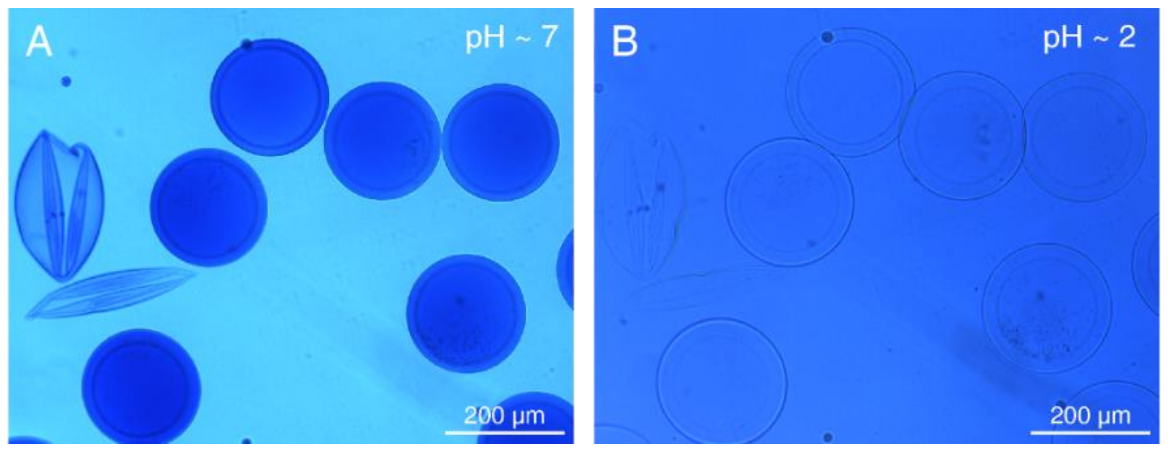

Figure S6: (A) Optical micrograph of PAA loaded capsules dispersed in a methylene blue containing solution at $(\mathrm{A}) \mathrm{pH} \sim 7$ and $(\mathrm{B})$ their release at $\mathrm{pH} \sim 2$. 